\title{
Apresentação
}

\section{E por falar em coleções e representação...}

\author{
Edmundo Pereira \\ Professor Doutor da Universidade Federal do Rio de \\ Janeiro/ Museu Nacional, Rio de Janeiro, Brasil \\ edmundopereira@gmail.com \\ Manuel Lima Filho \\ Professor Doutor da Universidade Federal de Goiás, Goiânia, Brasil. \\ manuellimafilho@gmail.com \\ “O fascínio de uma coleção está nesse \\ tanto que revela e nesse tanto que \\ esconde do impulso secreto que levou a \\ criá-la" (Calvino, 1974).
}

Apresentamos à comunidade acadêmica o dossiê "Coleções, Colecionadores e Práticas de Representação" da revista Sociedade e Cultura da Universidade Federal de Goiás. Fomos motivados, após conduzir e participar de fóruns e grupos de trabalho em encontros como os da Associação Brasileira de Antropologia (ABA) e da Associação Nacional de Pós-Graduação em Ciências Sociais (ANPOCS), a estimular a publicação de pesquisas no campo da antropologia e ciências sociais e suas interfaces disciplinares a respeito do tema da formação e gestão de coleções artístico-científicas e as práticas de representação a estas associadas, uma vez que se tem notado um aumento de interesse pelas temáticas fomentadas pela revisão e retomada críticas da antropologia com relação aos estudos da cultura material, dos museus e da formação de campos disciplinares e instituições artístico-científicas. ${ }^{1}$

1 Para citar alguns exemplos de investimentos em curso desde a virada das décadas de 1980-1990: Stocking Jr., 1985; Thomas, 1991; Cohn, 1996; O’Hanlon; Welsch, 2002; Findlen, 1994; Bennett, 1995; Bleichmar, 2012; Karp; Kreamer; Lavine, 1992; Abreu; Chagas, 2002; Ewbank; Gripp, 2016; Appadurai, 2008; Gell, 1998; Kopytoff, 2008; Abreu, 2005; Cury, 2006; Athias; Lima Filho, 2016; Velthem, 2003; Gonçalves, 2007; Oliveira, 2007; Pereira; Lima Filho, 2016. 
Como apontou Fabian (2010), depois dos efeitos e caminhos epistemológicos da associação entre poética e política para a escrita antropológica, os estudos de "cultura material" têm tido um papel crucial nas reorientações que a Antropologia vem assumindo num movimento mais consciente de superação do positivismo analítico e das implicações ético-políticas do trabalho científico frente à condenação moral que sofreu enquanto partícipe da empresa colonial-imperial. O papel da cultura material, das materialidades, suas técnicas, estéticas e éticas, é retomado tanto de um ponto de vista etnográficoanalítico, com o aparecimento de etnografias históricas dos processos de colecionamento e musealização em suas implicações para a organização de campos artístico-científicos e de administração colonial; quanto ético-moral com efeitos nas reflexões teóricas sobre os estatutos de distinção de coisas e objetos e a ampliação de noções como as de 'sujeito', 'biografia', 'vida'.

O 'objeto', muito mais do que um dado inerte nas reservas técnicas ou arquivado em nossos gabinetes de estudos, passa a ter repercussões e protagonismos, desta maneira, assumindo um estatuto polifônico e hermenêutico (Lima Filho, 2012) que conecta ações e ideias sobre 'arquivos', 'aquisição', 'conservação', 'curadoria', 'propriedade', 'natureza/ cultura', 'agência', 'arte', 'ciência' e atos de colecionar (Lima Filho, 2012b). Este quadro configura um campo de debates complexo, enredado e, não raro, tenso. Políticas de representação se reorientam e efeitos práticos de colecionamento, arquivo e exibição podem ser notados entre pesquisadores, museólogos, curadores e representantes de coletivos e povos historicamente colecionados e exibidos. Um caso que bem explicita essa nova orientação é o descrito por Oliveira (2007) sobre sua experiência museal, tendo como referência um objeto da reserva técnica do Museu Nacional associado ao povo Bororo:

as coleções etnográficas, uma vez retiradas das aldeias e dos seus contextos de utilização cotidiana e ritual, tendem a ser transformadas em abstrações sociológicas, nas quais a história e a reflexividade estão congeladas. A análise de uma pintura de um menino Bororo, localizada no Museu Nacional, permite reencontrar estórias esquecidas e encobertas, apresentando uma abordagem alternativa que propõe uma historicização radical e discute o jogo de forças que estão em torno da aquisição, classificação e exibição de objetos etnográficos (Oliveira, 2007, p. 73).

Dentro desse desenho geral de questões, autores e horizontes teóricos, ao propor e organizar o presente dossiê partimos da evidência de que a geração e a administração de 'coleções' organizam-se em práticas ligadas a múltiplos projetos: disciplinares, de desenvolvimento e autonomização de campos científicos e artísticos; de poder e governabilidade; de invenção e administração audiovisual da nação; e do império. Para dar conta desse quadro de investimentos etnográficos e analíticos, vem se definindo um conjunto de preocupações focadas, em particular, nos atos de colecionamento, ${ }^{2}$ no entre-

2 Veja: Thomas, 1991; Cohn, 1996; O’Hanlon; Welsch, 2000; Fabian, 2010; Findlen, 1994; Oliveira, 1987; Pereira, 2016a, 2016b; Lima Filho, 2017. 
debate sobre a formação de arquivos (Museus, Bibliotecas, Herbários, Jardins zoológico e botânico); na organização distintiva das Ciências e das Artes; e, recentemente, na geração de contrarrepresentações frente a regimes de subalternização (indexados em referentes como 'primitivo', 'tradicional', 'folclórico'). Esses investimentos, debruçados sobre economias simbólicas complexas, colocam em evidência a relação entre investigadores e interlocutores na objetificação da diversidade 'cultural' e 'natural' (de catálogos de plantas e acervos de herbários, até coleções de objetos e coletâneas de poemas, canções e melodias) com auxílio de múltiplas tecnologias audiovisuais de mediação da observação e da experiência.

O conjunto de trabalhos recebidos evidencia a vitalidade e o rendimento desse enquadre de investigação e análise, e apresenta um quadro amplo de cenários, personagens, projetos e temporalidades do campo das coleções e das práticas associadas aos processos de constituição, organização e exposição de bens artefatuais. ${ }^{3}$ Ao mesmo tempo, aponta para áreas de investigação como: (a) a formação de 'coleções científicas' no quadro geral da organização e associação das ciências naturais e da nação no século XIX; (b) a formação de coleções na organização das práticas etnográficas e da disciplinaridade da Etnologia indígena; (c) a musealização de coleções artístico-científicas e suas implicações para a subalternização e higienização de segmentos sociais das histórias da 'nação'e da 'civilização'; (d) a formação de coleções particulares, em quadro de colecionamentos populares, e o modo como se consagram como 'história local' ou 'biografia'; (e) a formação de coleções sonoro-musicais no quadro de associação entre tecnologias de registro, arquivo e difusão, representações fonográficas e identidades sonoras coletivas.

Abrimos com dois momentos da relação entre ciências naturais, nação e império ao longo do século XIX. No primeiro momento, através da trajetória colecionista do 'naturalista' Johann Naterrer, Rita Santos refaz alguns de seus percalços em solo brasileiro, nas primeiras décadas do século XIX, para a formação de coleções múltiplas: osteológicas, filológicas, de curiosidade. Através da montagem de algumas 'situações etnográficas', apresenta o modo como redes de conhecimento e administração são articuladas tanto do ponto de vista do modo como condições de colecionamentos são geradas quanto da longa cadeia de significação e esquecimentos pela qual coleções e colecionadores passam. Objetos etnográficos configuram-se em 'objetos de pacificação' tanto em sentido concreto, na grande rede de trocas de objetos que organiza os campos indigenistas, quanto imaginário, no lugar que certos povos e objetos vão ganhando no horizonte cultural da nação e de sua capacidade de administrar populações.

No caso das 'exposições nacionais' que povoam o último quartel da formação da nação, como em contexto americano, Danilo Duarte convida a pensar, através da recuperação dos processos de migração de 'europeus' ('alemães') para as américas no

3 Foram recebidas quase duas dezenas de propostas de publicação, representando um quadro abrangente de regiões, instituições e referenciais etnográfico-analíticos, o que se procurou manter, em menor grau, para a seleção reunida. Agradecemos ao conjunto de autores e autoras e lamentamos não poder publicar o total de trabalhos por questões de limitação editorial. 
final do XIX, os problemas de organização e difusão de patrimônios locais para geração de representações nacionais. Um mesmo processo colecionista pode congregar distintos projetos de marcação de civilidade em solo americano de gente que veio de longe (alemães no Chile); à 'riqueza na nação' botânica e mineral. Os problemas no entorno do que podemos chamar de cidadania patrimonial (Lima Filho, 2015) não vem de hoje, mas podem também ser encontrados nos modos coletivos, utilizando materiais locais muitas vezes pouco conhecidos para performar presenças longevas, coleções que representam presença, capacidade de trabalho, 'civilização' frente ao 'selvagem'.

Em seguida, adentramos pela relação entre a formação da etnologia indígena como campo disciplinar e a formação de coleções etnográficas de museus tanto de um ponto de vista de comparação de distintos momentos de prática de colecionamento quanto do modo como as críticas contemporâneas à formação e exibição de acervos etnográficos geraram formas alternativas de estabelecimento de relações articuladas entre indígenas e não indígenas na formação, conservação e exibição de artefatos. Para o caso das práticas de colecionamento de "objetos indígenas", no caso "karajá", grupo que apresenta longa história de colecionamento, Rafael Santana Gonçalves de Andrade chama nossa atenção para o caso das "máscaras rituais" do povo Karajá do rio Araguaia, provenientes da pesquisa do antropólogo estadunidense William Lipkind nos anos de 1938 e 1939 O estudo da coleção W. Lipkind do Museu Nacional/UFRJ conduziu o autor a comparar com outras duas situações etnográficas quando máscaras foram retiradas das aldeias: Paul Eherenrecih (1888) e Fritz Krause (1908). Aparentemente inertes, objetificadas pelo classificador "máscara", foram deslocadas de aldeias ao longo de gerações como itens 'etnográficos', ainda que fosse a prática considerada pelo grupo como violação do sagrado, profanação, uma vez serem estas relacionadas aos segredos dos homens. Prática de horizonte colonialista que, se recuperada ao rés das relações sociais, revelam suas ambivalências e contradições entre 'objetos rituais' e 'antepassados', colocando em pauta, no presente, na retomada do tema da 'cultura material' na Antropologia e áreas afins, não só questões de políticas de representação exibicionária, mas da revisão de protocolos da pesquisa de campo e gestão museológica.

No campo dos museus e da 'mediação cultural' de processos dialógicos e participativos, no quadro das reações científico-museais às críticas indígenas, de reposicionamentos e busca de práticas alternativas, temos a experiência da museologia-antropologia compartilhada relata por Adriana Russi com um grupo indígena amazônico, os Katxuyana, e o reencontro por meio de fotografias com artefatos do grupo acervados em museus de longa tradição em colecionamento e exibição: o Museu Nacional, no Rio de Janeiro; Museu Paraense Emílio Goeldi, em Belém; British Museum, em Londres; Kulturhistorisk Museum, em Oslo/Noruega; Museum für Völkerkunde, em Hamburg/Alemanha; e Moesgaard Museum, em Arhus/Dinamarca. $\mathrm{O}$ acesso às imagens provocou um debate entre jovens e velhos das aldeias conformando campos de reflexão em que condições de colecionamento foram rememoradas e propostas contemporâneas foram organizadas. 
Aos olhos do presente, como exemplo de um antimuseu, a recuperação de Clóvis Brito da musealização de restos mortais do 'cangaço', a partir da trajetória das 'cabeças' de Maria Bonita e Lampião no Museu-Casa de Maria Bonita, em Malhada da Caiçara, Paulo Afonso-BA, revela tanto sua potencialidade de índice historiográfico das relações sociais em que se organizava parte do nordeste brasileiro das primeiras décadas do século XX quanto de subalternização post mortem, com geração de 'despojos', em especial 'cabeças', exibição pública e posterior conservação e exibição museais. O processo de musealização desses remanescentes humanos equaciona de maneira acrítica e inesperada sua exibição como troféu de guerra, prova de crime e objeto científico, atos de colecionar e exibir que perpassam e configuram debates sobre ética, violência e poder.

Os problemas das disputas que envolvem a objetificação museal de certos conjuntos e temáticas reaparecem, desta vez no exercício comparativo Brasil-Estados Unidos de Marina Roriz, quando o conceito de arte e a experiência estética dos visitantes de certos circuitos museais são hipervalorizados a desfavor de certo contexto social cultural mais amplo, nacional e local, no caso em particular de higienização nas coleções exibidas da presença de grupos étnico-raciais, como é o caso dos 'afro-americanos' em Inhotim, Minas Gerais e em Saint Louis, Missouri. Supervaloriza-se a contemplação estética e silencia-se a história de alguns grupos e personagens, transformando-a em narrativa e produto.

No trabalho de José Rogério Lopes e Rodrigo Manoel Dias da Silva, somos levados a outros níveis e redes de colecionamento, não mais em contextos de institucionalização e profissionalização artístico-científica, mas de produção de certas culturas populares de colecionamento e memorialismo, de formação de 'arquivos pessoais'. Desta forma, convidam, inicialmente, a ampliar os 'quadros da experiência colecionista' para trajetórias individuais diversificadas, e o espectro de materiais colecionáveis, como 'histórias', 'casos' e 'acontecimentos' e fotografias. E, depois, para a maneira como práticas de colecionamento particular podem se institucionalizar sendo incorporadas à memória social local. Cidadãos patrimoniais de redes domésticas (como as de Canela, RS, e São Luiz do Paratininga, SP) que, em alguns casos, a depender da coleção, das redes de especialização de conhecimento e intercâmbio de objetos e informação, podem ultrapassar essas dimensões adentrado regime de registro de localidade, de região em redes que articulam projetos memorialistas pessoais e de estado.

Por fim, no contexto da geração de coleções sonoro-musicais, fechamos com o exercício de Sabrina Dinola, Regina Abreu e Amir Geiger, ocupados com os temas e questões que a geração e gestão de coleções podem fomentar através da comparação de alguns períodos de colecionamento sonoro, associando objetificações sonoras da cultura com contextos tecnológicos de registro e difusão. A ênfase inicial nas formas de registro sonoro e nas representações fonográficas geradas salienta o caráter 'atuante' de 'objetos musicais' como 'fonogramas', conformando imaginações musicais em quadro que se pretende da 'região' e da 'nação'. Comparando períodos - dos iniciais, entre as décadas de 1910 e 1930 com os trabalhos de Roquette-Pinto e Mário de Andrade, às 
'retomadas' na década de 1980 com enfoque etnomusicológico, e depois em quadro de indústria cultural e, por fim, digital -, estes enfatizam tanto continuidades nos processos de formação de registros fonográficos associados a questões de identidade cultural quanto as novas configurações e dilemas que o mundo digital traz, em quadros complexos de políticas de representação sonora e disputas por direitos patrimoniais.

Alinhavando esta coleção de objetos, personagens, cenários e esforços etnográficoanalíticos, enfatizamos que a eleição dos referentes coleções, colecionadores e práticas de representação como fios condutores objetiva agregar investimentos referenciais de forma a dar conta das condições de geração de conhecimento artístico-científico em contextos de definição disciplinar e profissional, e suas associações com políticas da memória, da representação e da administração de bens culturais. Em alguma medida, esperamos, editores, autores e autoras, que o dossiê ajude a revelar campos temáticos que apresentem a pluralidade e a complexidade de feixes de configuração que envolve os atos de colecionar e a gestão de coleções ao longo das trajetórias de objetificação das pessoas e das coisas, dos conhecimentos, regiões, povos e coletivos a estes associados.

$* * *$

\section{Referências}

ABREU, Regina. Museus etnográficos e práticas de colecionamento: antropofagia dos sentidos. Revista do Patrimônio Histórico e Artístico Nacional, Rio de Janeiro, v. 31, p. 100-125, 2005.

APPADURAI, Arjun. (Org.). A vida social das coisas: as mercadorias sob uma perspectiva cultural. Niterói, RJ: Editora da Universidade Federal Fluminense, 2008.

ATHIAS, Renato; LIMA FILHO, Manuel F. Dos museus etnográficos às etnografias dos museus: o lugar da antropologia na contemporaneidade. In: RIAL, Carmen; SCHWADE, Elisete (Org.). Diálogos antropológicos contemporâneos. Rio de Janeiro: ABA, 2016. p. 71-83.

BENNETT, Tony. The Birth of the Museum. History, Theory and Politics. New York: Routledge, 1995.

BLEICHMAR, Daniela. Visible Empire. Botanical Expeditions \& Visual Culture in the Hispanic Enlightment. USA:The University of Chicago Press, 2012.

CALVINO, Italo. Coleção de Areia. São Paulo: Companhia das Letras, 2010.

COHN, Bernard. Colonialism and Its Forms of Knowledge. USA: Princeton University Press, 1996.

CURY, Marilia Xavier (Org.). Direitos indígenas no museu: novos procedimentos para uma nova política - a gestão de acervos em discussão. São Paulo: USP; Brodowski: MAE, 2016.

EWBANK, Cecilia de Oliveira; GRIPP, Maria Pierro. O oculto em movimento: ressignificando uma coleção etnográfica na reserva técnica. Anais da 30 Reunião Brasileira de Antropologia, 2016. Disponível em: <http:// www.30rba.abant.org.br/arquivo/downloadpublic?q=YToyOntzOjY6InBhcmFtcyI7czozNToiYToxOntzOjEwOiJJRF9BUlFVSVZPIjtzOjQ6IjI4MzUiO30iO3M6MToiaCI7czozMjoiMDAyZDY2ODI5YjE3NDlkOWVlYzE5OGE5YjJkZWNhMmUiO30\%3D>. Acesso em: 27 jan. 2018.

FABIAN, Johannes. Colecionando pensamentos: sobre os atos de colecionar. Mana, v. 16, n. 1, p. 59-73, abr. 2010.

FINDLEN, Paula. Possessing Nature. Museums, Collecting and Scientific Culture in Early Modern Italy. USA: University of California Press, 1994.

GELL, Alfred. Art and agency: an anthropological theory. New York; London: Clarendon Press, 1998. 
GONÇALVES, José Reginaldo Santos. Antropologia dos Objetos. Coleções, Museus e Patrimônios. Rio de Janeiro: Garamind; Minc/IPHAN, 2007.

KARP, Ivan; KREAMER, Christine Mullen; LEVINE, Steven (Ed.). Museums and Communities. The Politics of Public Culture. USA: Smithsonian Institute, 1992.

KOPYTOFF, Igor. A biografia cultural das coisas: a mercantilização como processo. In: APPADURAI, Arjun. (Org.). A vida social das coisas: as mercadorias sob uma perspectiva cultural. Niterói, RJ: Editora da Universidade Federal Fluminense, 2008.

LIMA FILHO, Manoel Ferreira. Cidadania patrimonial. Anthropológicas, Rio de Janeiro, ano 19, v. 16, n. 2, p. 134$155,2015$.

LIMA FILHO, Manuel Ferreira. Coleção William Lipkind do Museu Nacional - rotas antropológicas BrasilEstados Unidos. Mana, Rio de Janeiro, v. 23, n. 3, p. 473-507, set./dez. 2017.

LIMA FILHO, Manuel Ferreira. Cultura Material e saberes antropológico. In: LIMA FILHO, Manuel Ferreira; TAMASO, Izabela Maria. (Org.). Antropologia e Patrimônio Cultural: trajetórias, conceitos e desafios. Goiânia: Cânone Editorial, 2012.

LIMA FILHO, Manuel Ferreira; ABREU, Regina; ATHIAS, Renato (Org.). Museus e Atores Sociais: perspectivas antropológicas. Recife: Ed. UFPE, 2016.

O'HANLON, Michael; WELSCH, Robert (Ed.) Hunting the gatherers. Ethnographic Collectors, agents and agency in Melanesia, 1870-1930s. USA: Bergahn Books, 2002.

OLIVEIRA, João Pacheco de. Elementos para uma sociologia dos viajantes. In: Oliveira, João Pacheco de (Org.) Sociedades Indígenas e Indigenismo no Brasil. Rio de Janeiro: UFRJ, Ed. Marco Zero, 1987.

OLIVEIRA, João Pacheco de. O retrato de um menino Bororo: narrativas sobre o destino dos índios e o horizonte político dos museus, séculos XIX e XXI. Tempo, Niterói, RJ, v. 12, n. 23, p. 73-99, 2007. http://dx.doi.org/10.1590/S1413-77042007000200006.

PEREIRA, Edmundo; LIMA FILHO, Manuel Ferreira. 2016. Desnaturalizando assimetrias: o "outro" dentro do Museu. In: Trabalho apresentado no VI Congresso da Associação Portuguesa de Antropologia. Portugal: Coimbra, 2016.

PEREIRA, Edmundo. Notas sobre representação fonográfica, ritual de gravação e tradição musical. In: LIMA FILHO, Manuel Ferreira; ABREU, Regina; ATHIAS, Renato (Org.) Museus e Atores Sociais: perspectivas antropológicas. Recife: Editora UFPE, 2016a. p. 215-244.

PEREIRA, Edmundo. Representação fonográfica e curadoria sonora: notas sobre dialogia e desentendimento. In: XAVIER, Marília Cury (Org.). Direitos indígenas no Museu: novos procedimentos para uma nova política: a gestão de acervos em discussão. São Paulo: Secretaria da Cultura; ACAM Portinari; Museu de Arqueologia e Etnologia da Universidade de São Paulo, 2016b.

STOCKING Jr., George. (Ed). Objects and Others. Essays on Museums and Material Culture. USA:The University of Wisconsin Press, 1985.

THOMAS, Nicholas. Entangled Objects. Exchange, Material Culture and Colonialism in the Pacific. UK: Harvard University Press, 1991.

VELTHEM, Lucia Hussak van. Objets de memóire: indiens, colletions et musées au Brésil. i, n. 45, p. 133$149,2003$. 